\title{
A convertible spinal orthosis for controlled torso rigidity
}

\author{
Nicole I. Kern ${ }^{\mathrm{a}, *}$, Ronald J. Triolo ${ }^{\mathrm{b}, \mathrm{c}, \mathrm{d}}$, Rudi Kobetic ${ }^{\mathrm{e}}$, Musa Audu ${ }^{\mathrm{e}, \mathrm{f}}$ and Roger D. Quinn ${ }^{\mathrm{a}}$ \\ ${ }^{a}$ Department of Mechanical Engineering, Case Western Reserve University, Cleveland, OH, USA \\ ${ }^{\mathrm{b}}$ Department of Orthopaedics, Case Western Reserve University, Cleveland, OH, USA \\ ${ }^{\mathrm{c}}$ MetroHealth Medical Center, Cleveland, OH, USA \\ ${ }^{\mathrm{d}}$ Advanced Platform Technology (APT) Center, Louis Stokes Cleveland Veterans Affairs Medical Center, \\ Cleveland, $\mathrm{OH}, \mathrm{USA}$ \\ e FES Center, Louis Stokes Cleveland Veterans Affairs Medical Center, Cleveland, OH, USA \\ ${ }^{\mathrm{f}}$ Department of Biomedical Engineering, Case Western Reserve University, Cleveland, OH, USA
}

\begin{abstract}
A traditional spinal orthosis in conjunction with a hip-knee-ankle-foot orthosis (HKAFO) improves posture in persons with paraplegia during standing and walking. It also limits the wearer's range of motion when worn during other activities, such as vehicle transfer or sitting and reaching for objects. In order to regain full torso flexibility the user would need to remove the spinal orthosis which can be arduous and time consuming. A Convertible Spinal Orthosis (CSO) that allows the user to switch between Locked rigid torso support and Unlocked free motion has been designed, fabricated and tested. It shows promise for increasing functionality, wear time and subject comfort. Analysis of movement has been performed with an able-bodied and a paraplegic subject wearing a rigid spinal orthosis, the CSO in both states, and without any bracing. Configuration state had the most impact on lateral bending. Mean values for the paraplegic subject of $27^{\circ}, 38^{\circ}, 48^{\circ}$, and $48^{\circ}$ and for the able-bodied subject of $22^{\circ}, 26^{\circ}, 48^{\circ}$, and $45^{\circ}$ were found for lateral bending of the upper torso relative to the thighs in the Rigid, Locked, Unlocked, and No-Brace states, respectively.
\end{abstract}

Keywords: Hybrid neuroprosthesis, spinal orthosis, compliant trunk mechanism, compliant spinal orthosis

\section{Introduction}

A hybrid neuroprosthesis (HNP), comprising both functional neuromuscular stimulation (FNS) and a mechanical bracing system, has been developed with the aim of overcoming mobility and accessibility limitations inherent in paraplegia [10]. Motor function is achieved through electrical stimulation of paralyzed muscles and synchronized for multi-joint actions such as walking. Stimulation of paralyzed muscles can successfully propel the body forward, however, walking

\footnotetext{
*Corresponding author: Nicole I. Kern, Department of Mechanical Engineering, Case Western Reserve University, 10900 Euclid Ave, Cleveland, OH, USA. Tel.: +216 368 5216; Fax: +216 368 6445; E-mail: nicole.kern@case.edu.
}

with FNS alone is characterized by excessive forward lean and upper extremity effort, as well as rapid fatigue from the sustained stimulation required to support body weight [12]. In contrast, bracing systems provide upright posture and safety against collapse through a mechanical support structure. The HNP is designed to combine the power of FNS with the support of a bracing system.

Traditional orthotic systems lock the lower limb joints for stability, though this leads to unnatural gait and walking with immobilized joints requires more energy $[6,14]$. As an alternative to traditional bracing, the HNP uses a low-power control and actuation scheme to allow movement of the torso as well as the hips, knees, and ankles when not supporting body 
weight. Mechanisms at these joints are timed to secure the body against collapse during support phases of gait or when instability is sensed. In using the HNP the body is driven by FNS and is stabilized by the bracing system, in an effort to restore sustainable and safe bipedal locomotion to individuals paralyzed by spinal cord injury (SCI) $[9,13]$.

Mobility systems for persons with disabilities are typically improved with use of a walking aid to preserve equilibrium, support weight, change direction, or maintain balance. With orthotic systems in particular it can be difficult to maintain trunk posture and therefore equilibrium, even with the use of a walking aid [8]. A rigid spinal orthosis, such as a thoraco-lumbosacral-orthosis (TLSO), would reinforce the function of any existing core musculature and maintain stable posture. However, conventional spinal orthoses restrict torso motion at all times, making activities other than walking and standing difficult or uncomfortable unless the bracing system is removed. For optimal function, the orthosis should be capable of either providing rigid support or allowing free movement of the torso. This is the basis for the Convertible Spinal Orthosis (CSO), which has been developed as part of the HNP project. The CSO is actuated so that the wearer may switch between the unlocked state of free movement (Fig. 1) and the locked support state.

\section{Related work}

Previous work on hybrid neuroprostheses has produced several systems that use FNS as a sole driving force in combination with energetically inert elements to affect motion. These include the CBO (Controlled Brake Orthosis) [3] and JCO (Joint Controlled Orthosis) [2], which are driven by surface stimulation of the quadriceps muscle group of each leg. Spring assist and mechanical joint coupling aid movement while friction brakes damp motion to maintain a desired limb trajectory. The SBO (Spring Brake Orthosis) [4] uses surface stimulation with a tension spring across the knee to store energy and a wrapped spring clutch to brake joint movement at the hip and knee. Both pneumatic cylinders and rubber bands are used as energy storage devices in the ESO (Energy Storing Orthosis) [5], with wrap spring clutches at the hip and knee for braking. Hybrid systems generally focus on motion of the lower limb joints, especially the hip and knee.

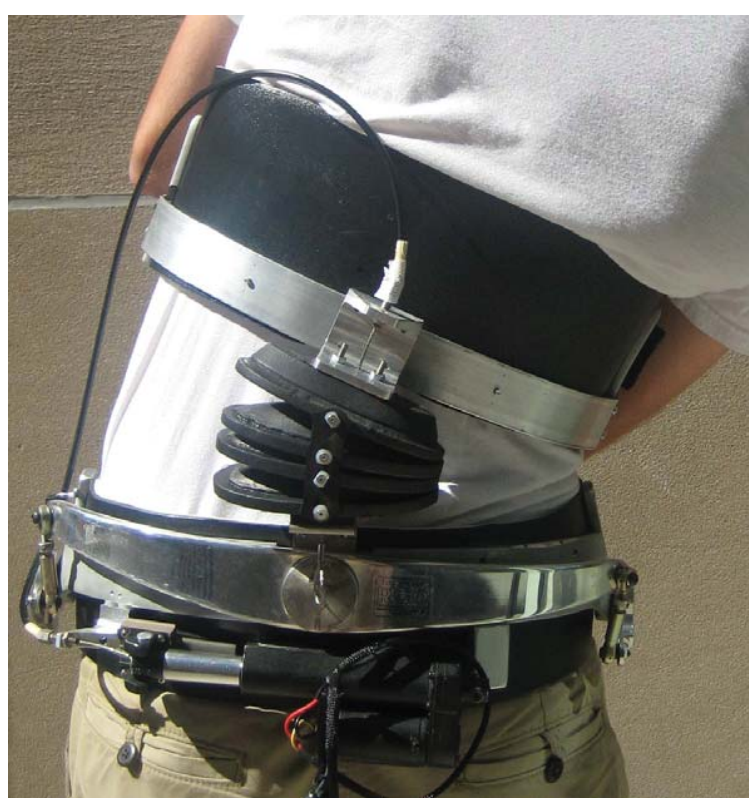

Fig. 1. A user bending laterally while wearing the CSO in the unlocked configuration.

In order to better understand the role of the spine in able-bodied walking, Konz, Fatone and Gard investigated the effect of spinal restriction on gait in able-bodied persons with and without a fiberglass body jacket similar to a TLS O [11]. With the spine restricted, hip ab/adduction and pelvic rotation were significantly decreased at all walking speeds, and there was an increase in hip flexion and extension at slow speeds. In another study, Zhang et al. set out to determine the efficacy of one lumbar and one lumbosacral orthosis by measuring the able-bodied range of motion (ROM) as compared with no bracing [17]. Maximum forward flexion was reduced for both orthoses, though axial twisting and lateral bending were not tested.

Cholewicki et al. measured and compared spine motion restriction and passive trunk stiffness provided by three commercially available TLSOs [1]. Using an EMG model to measure stiffness and a Flexducer strain gauge to measure flexion/extension and lateral bending, the group found no significant differences between the three TLSOs in any measure except perceived comfort. The measurement equipment was calibrated only for comparative angles, and no aggregate bending amounts were reported. A device called the split collar locking mechanism was previously designed in conjunction with the HNP. It was composed of a string of ball joints surrounded by attached 
overlapping collars [7]. Locking was achieved by sliding each set of collars against one another to force compression and prevent bending. The split collar locking mechanism was low-profile and each ball joint had a similar ROM to that between human vertebrae, but there was no simple way to attach the mechanism, allow translation, or control the lock state of each joint, especially not with a single actuator.

\section{Mechanism design}

\subsection{Specifications}

In its compliant state the CSO should allow considerable ROM in front/rear bending, lateral bending, and axial twisting. Since the locking mechanism is not coincident with the rotation axes of the spinal column, the device must also allow a fourth degree of freedom (DOF) in translation for bending to be possible. Locking must be actuated in such a way that the wearer can easily control the state of the device. In the interest of minimizing weight and power requirements, control of all four DOF should be achieved using one actuator. The mechanism should be safe, low profile, comfortable, and simple to doff and don. To ease system integration, the CSO should be compatible with existing HKAFO leg uprights. Ideally rear attachments should be low profile, or avoided altogether, so that the wearer could comfortably lean against a chair back.

\subsection{Prototypes}

The CSO mechanism is based on the use of lockable columns to control rigidity. A cable is pulled to achieve the locked state, which compresses the stack of plates tightly enough to act as a solid column between top and bottom sections of the orthosis (Fig. 2). The top and bottom plates are fixed to their respective brackets on the upper and lower portions of orthosis, and the middle plates are free when the stack is uncompressed. Multiple prototypes were built by modifying the thoracic jacket of a commercially available reciprocal gait orthosis (RGO) with lower leg components intact for ease of integration. The middle section of the spinal orthosis was cut away and replaced with one or more columns of stacked plates or discs to form the CSO.

The first prototype (Fig. 3) had four columns of eight round acetal spacers with central holes for each of four cables. Locking was controlled manually through four

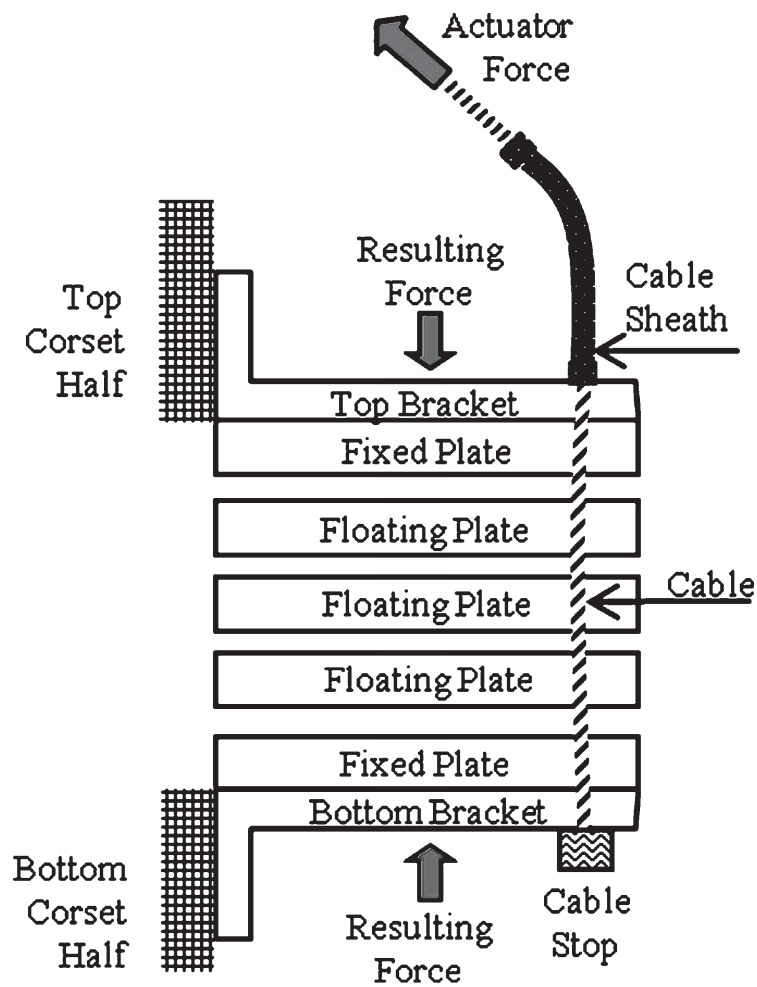

Fig. 2. Side cut-away showing how the actuator force is transferred to compress the stack and enforce rigidity.

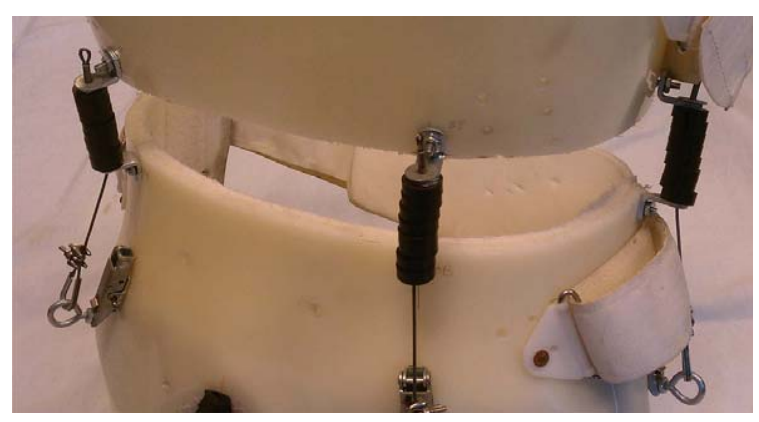

Fig. 3. First prototype with four manually actuated latched columns

individual over center latches, with both lock-tabs and cotter pin holes for security. This prototype was tested with an able-bodied user and successfully constrained bending and twisting while locked, and allowed free motion while unlocked. However, it was determined that a user with SCI would have difficulty reaching and closing each latch, and therefore electrical actuation became a requirement. 


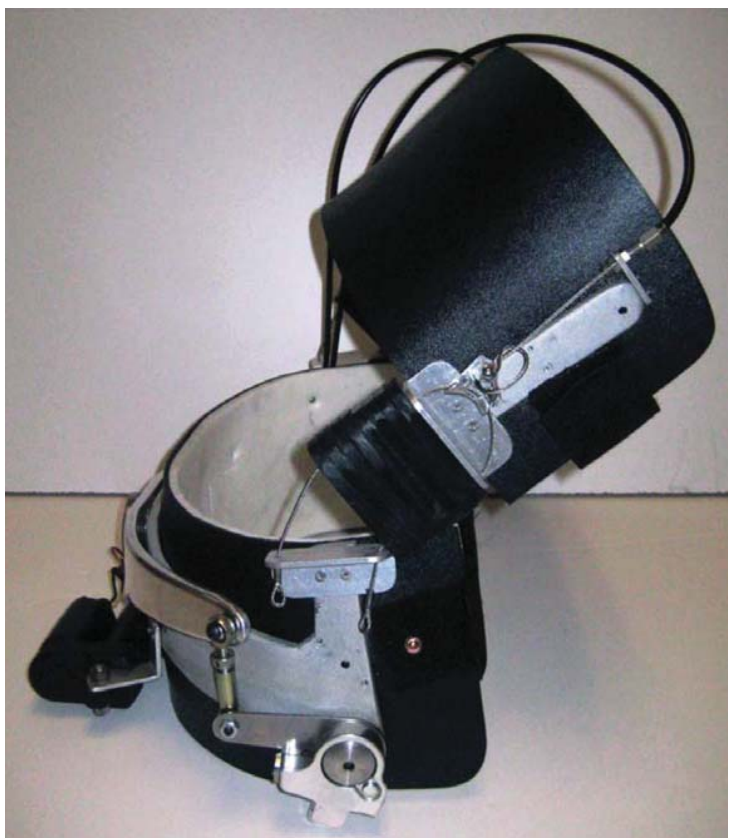

Fig. 4. Second prototype with two columns of flat plates at the sides.

Using a cable attached to the moving end of a linear actuator the control methodology is simple. When the end is extended there is slack in the cable and the CSO is unlocked, and when the end is retracted the cable is tensioned and the CSO is locked. The Electrak ${ }^{\circledR} 1$ (Warner Electric, South Beloit IL, USA) with limit switches, $25 \mathrm{lb}(111 \mathrm{~N})$ force capacity and $2^{\prime \prime}(51 \mathrm{~mm})$ stroke was chosen for its size and affordability.

A second prototype was built with two stacks of acetal plates, one at each side (Fig. 4). Experimentation with an able-bodied subject was performed using the CSO connected to a traditional RGO with the reciprocator disengaged allowing free motion at the hips. The subject walked at a comfortable speed in locked and unlocked configurations, with unannounced changes from one state to the other. Switching from the locked to the unlocked configuration was repeatedly successful. However, the mechanism would not fully lock if there was already a length of free cable squeezed between two plates, as can occur in the unlocked configuration with the plates out of alignment from the wearer twisting to on e side (Fig. 5).

To avoid cable friction between plates, multiple designs consisting of both interlocking and nested shapes were considered. Though interlocking parts provide the best protection against cable pinching (Fig. 6), they are also more difficult to manufacture.

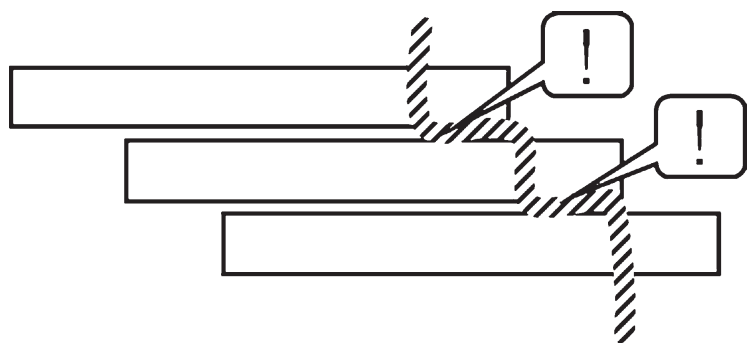

Fig. 5. An illustration of a pinched cable between misaligned plates.

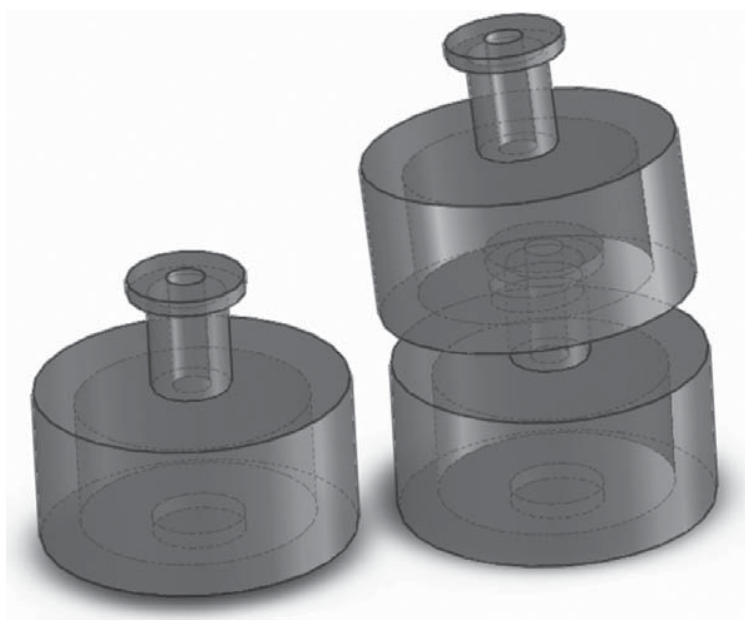

Fig. 6. An example of an interlocking shape design for cable routing.

Some nested shape designs were found to be commercially available. Plastic cones were chosen as the basis for the next stack material.

Each column of plates was replaced with two columns of nested cones. The cones had a central hole for cable routing, and the maximum spacing between consecutive cones was controlled by a fixed length of chain (Fig. 7). The chain was important for preventing the phenomenon illustrated in Fig. 5. Since the chain length was chosen so that the cable was constrained from leaving the bottom of each cone before being threaded through the top of the next cone, the cable could not be crushed during locking. The design produced fair results; the cones often stuck together preventing full unlocking, which could have been prevented by sourcing or fabricating thicker cones with a less shallow slope. There was also no guarantee that the two cables on each side would be pulled with the exact same force, which could lead to bias towards the front or back when locked. A design with a single cable would be more elegant. 


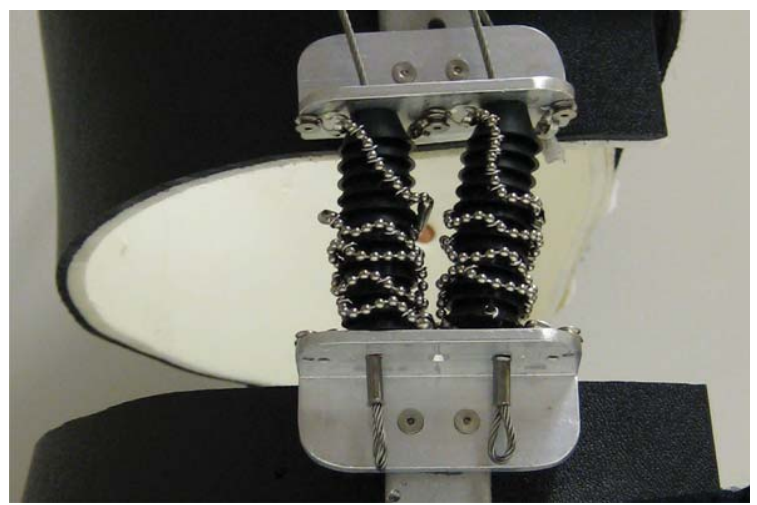

Fig. 7. Fitted cones with chains to limit travel. The cone shape guides the cable to prevent pinching between mating surfaces during locking.

A critical weakness with the side plate design became obvious after the cable pinching issue was resolved. In order for the wearer to flex both hips and move into a seated position, the hip reciprocator must be uncoupled, so that the hips can flex independently. This allows for a large range of front bending at the hips regardless of the CSO configuration. However, since the hips are constrained laterally, a significant range of lateral bending of the CSO is paramount to successful device function. In the cone prototype the ROM in front bending was comparable to able-bodied motion, but the ROM was severely decreased in both axial twisting and lateral bending, allowing less than $10^{\circ}$ of lateral bending from vertical. As an alternative to increasing actuator stroke length to provide more bending range, a new prototype was designed to address the issues of the cones sticking, the lack of force control in each individual cable, and the minimal ROM in twisting and lateral bending.

To increase ROM, a single stack of five acetal plates was used. This stack (Fig. 8) was fastened at the rear of the orthosis in alignment with the spine. A strip of aluminum was bent to conform to the top section of the orthosis for use as an attachment point. As a solution to both the cable-pinching and sticking-cones problems, each circular plate was designed with a flat edge with tapped holes for attachment to an elastomeric material. The elastic is compliant to allow free motion in the unlocked configuration, but provides a restoring force so that the plates self-align as the device is locked. This prototype provided increased ROM, however the ability to securely lock rotation was diminished since friction was the only mechanism preventing the plates

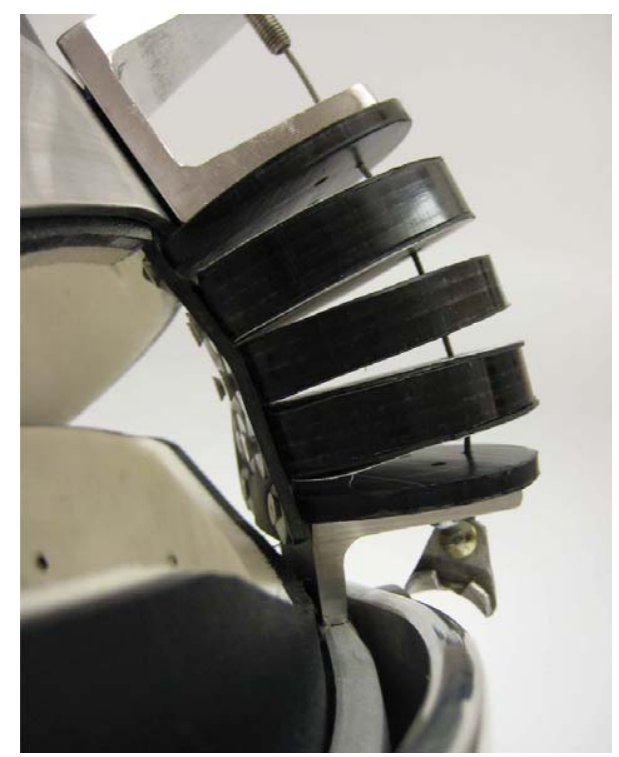

Fig. 8. First rear single stack design in the unlocked configuration.

from rotating relative to one another around the single cable.

To lock against axial twisting of the torso, the flat plates were replaced with a stack of five 3D kidneyshaped plates machined from acetal (Fig. 9). The rotationally asymmetrical plates nest inside of one another to provide a mechanical stop against twisting when compressed. Depending on the geometry of the plates, this design would allow for different amounts of lateral and front bending. For this prototype, the plates are shorter front to back so that the device has a low rear profile and the user is able to lean back, for example while seated in a wheelchair. An elastic material was again used to aid alignment during locking.

\section{Analysis}

\subsection{Work volume calculation}

The geometric relationship between plate dimensions, cable slack, and bending angle, as depicted in Fig. 10, can be described by Equation 1. An increase in slack $s$ and a decrease in radius $r$ leads to an increase in angle $\alpha$. Plate dimensions for length, width, and height were chosen to be $133 \mathrm{~mm}\left(51 / 4^{\prime \prime}\right), 64 \mathrm{~mm}$ (2 $\left.1 / 2^{\prime \prime}\right)$, and $6.4 \mathrm{~mm}\left(1 / 4^{\prime \prime}\right)$, respectively. These measurements were selected as a compromise between range and stability, since a smaller plate footprint and radius 


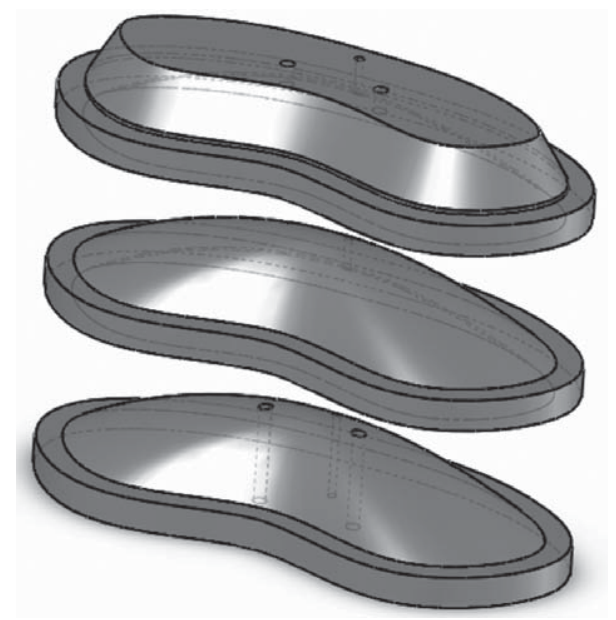

Fig. 9. Nested stack of plates designed to lock against rotation when compressed. The top and bottom plates are secured to their respective brackets, and the middle plates (only one shown) are free to rotate.

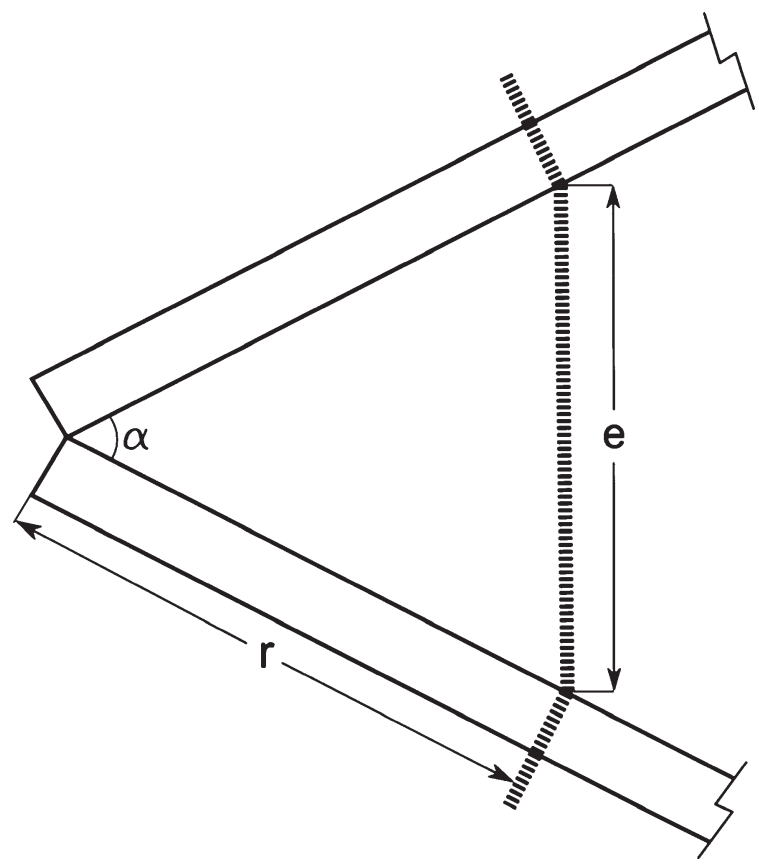

Fig. 10. Angle " $\alpha$ " relative to cable slack " $s$ " at radius " $r$ " from edge.

would allow more ROM, but would be more difficult to lock accurately. The maximum cable slack is determined by the actuator travel, which for the given actuator is $51 \mathrm{~mm}$. Since the cable runs through the middle of each plate, the radius for each direction is half of the plate dimension.
In this device axial twisting is limited only by the elastic restoring force once the plates are fully separated. The elastomer attachment to each plate promotes equal bending between each set of plates, and the total bending angle would be the sum of these individual angles. Assuming equal slack dispersion between each of the three plate gaps, the calculated angles for lateral bending (along plate length) and front bending (along plate width) are $44^{\circ}$ and $93^{\circ}$, respectively, according to Equation 1. Both of these values for bending of the plate mechanism could differ significantly from the bending angles of a subject inside the CSO for multiple reasons. For example, the subject may not be capable of reaching these angles even without bracing, the CSO could constrain movement in a way that limits subject ROM, or the CSO could interfere with itself.

$$
\sin (\alpha / 2)=s /(2 r)
$$

\subsection{Bench testing}

Bench testing was performed to verify that sufficient ROM was present in the CSO as a whole. Passive ROM was achieved by holding the bottom portion of the CSO stationary and moving the top portion by hand just until cable tension was felt. The top portion of the brace was held at this point and measured relative to the bottom portion with a protractor. The resulting ROM $-22^{\circ}$ of front bending, $25^{\circ}$ of lateral bending, and $35^{\circ}$ of axial twisting - were considered a sufficient improvement over the side plate prototype. The Locked state was estimated to be satisfactory after no plate movement was visually detected when the CSO was held from the top in different configurations with the weight of the bottom portion acting along each of the three principal directions.

\subsection{Quantifying movement}

The function of the CSO is straightforward in that ROM in four directions is locked or unlocked by a single actuator. In contrast, this actuation scheme leads to difficulty in quantifying the amount of bending that occurs in each direction. There are essentially no constraints on relative movement between the top and bottom sections of the orthosis, and bending often occurs in multiple directions simultaneously. Even when the cable slack is fully absorbed by the mechanism and a maximum ROM is reached, rotation can 


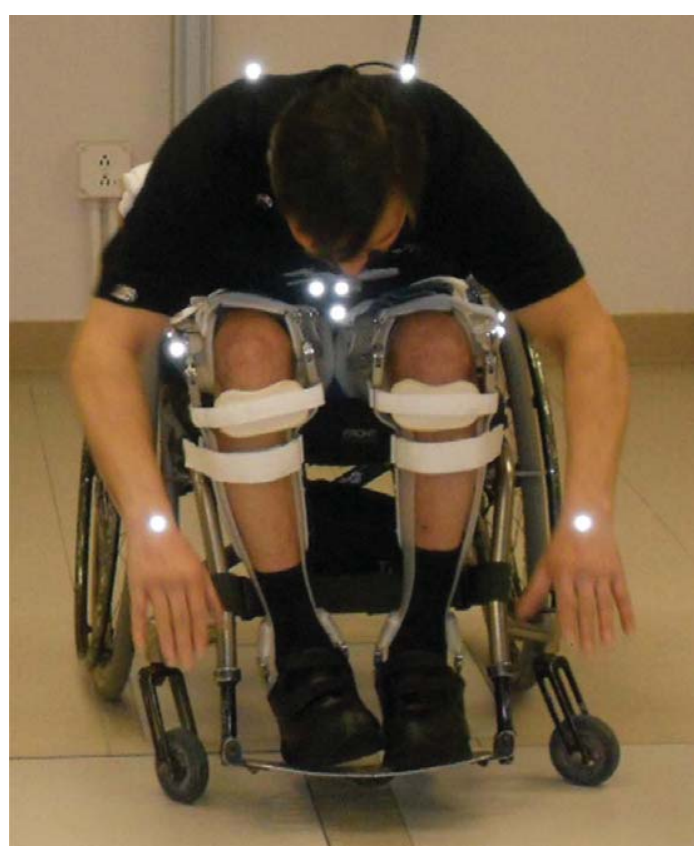

Fig. 11. Subject in Downward Flexion while wearing the unlocked CSO.

still occur in any combination of the three principal directions.

Multiple methods of quantifying motion were considered in order to ascertain the efficacy of the device. Accelerometers can be used to collect bending measurements, as verified in a study performed by Wong and Wong [15]. They showed that results from triaxial accelerometer measurements of trunk flexion and lateral bending could be accurate to within $5^{\circ}$. Wunderlich et al. recently developed a measurement system using an inclinometer and ultrasound measurements to calculate spinal motion in bending and axial twist [16], and they state that there is currently no "gold standard" to assess spine movements, though they mention 3D motion capture systems as a viable option. Ultimately, for this study a 16-camera Vicon MX40 motion analysis system (Vicon, Oxford, UK) was used to track the $3 \mathrm{D}$ coordinates of reflective markers placed on the subjects and orthosis.

\subsection{Data capture protocol}

Evaluations were performed with one male ablebodied subject and one male subject with T-7 complete SCI (ASIA A). For each of the two subjects, the procedure began with an explanation of each expected movement, with movements chosen to be representative of common activities of daily living. Each subject acted as their own concurrent control, so that each movement could be compared across the different interventions for each subject. All movements were performed while seated in a wheelchair (Fig. 11) or other surface at a height in which a $90^{\circ}$ bend was present at the knees with the feet flat. At the beginning of each trial the subject was asked to sit up as straight as possible, and then reach as far as possible, safely and comfortably, attempting to remain within the principal direction for each movement:

- Lateral Bend: bend to the side in the frontal plane as if to reach for an object next to you on the ground.

- Axial Twist: rotate along the transverse plane as far as possible as if to attempt to look at something behind you.

- Downward Flexion: bend forward towards the ground, as if to reach for an object on the floor in front of you.

- Forward Flexion: bend forward and out, as if to pull on a door knob or reach for an object at shoulder height.

A post hoc power analysis was performed on initial ROM data from these four movements to calculate sample size. A minimum sample size of four trials per subject, per configuration, was found to be sufficient for the Lateral Bend, Axial Twist, and Downward Flexion motions with a significance criterion of $p=0.01$. A minimum of six trials were required for the Forward Flexion movement to achieve the same statistical significance. Each movement was carried out with the subject in one of four different configurations:

- R: a Rigid, traditional spinal orthosis

- L: the Locked state of the CSO

- U: the Unlocked state the CSO

- $\mathbf{N}$ : No Brace; free

Both the rigid spinal orthosis and the CSO were worn in combination with an uncoupled RGO and leg uprights. All trials for each of the four configurations were performed before moving to the next configuration. Axial Twist and Lateral Bend were randomly assigned a left or right direction, and the order of all movements was randomized for each configuration. Movements were repeated a minimum of four times, or six times for bending forward, for a minimum of 18 trials per configuration and 72 trials per subject. 
Table 1

Body landmarks used to place markers for each segment

\begin{tabular}{|c|c|c|}
\hline & Bracing Trials & No Bracing \\
\hline Upper Torso & $\begin{array}{l}\text { L\&R Acromion, C7 } \\
\text { Vertebra, Clavicle }\end{array}$ & $\begin{array}{l}\text { L\&R Acromion, C7 } \\
\text { Vertebra, Clavicle }\end{array}$ \\
\hline Orthosis & $\begin{array}{l}\text { Any } 3 \text { Nonlinear Points } \\
\text { on Rigid Orthosis or } \\
\text { CSO Top Portion }\end{array}$ & $\begin{array}{l}\text { T10 Vertebra, L\&R } \\
\text { Lowest Rib at Rear }\end{array}$ \\
\hline CSO Bottom & $\begin{array}{l}\text { Reciprocating bar } \\
\text { center and L and R } \\
\text { Hip Joints of the RGO }\end{array}$ & N/A \\
\hline Thighs & $\begin{array}{l}\text { L\&R Greater } \\
\text { Trochanter and } \\
\text { Lateral Epicondyle }\end{array}$ & $\begin{array}{l}\text { L\&R Greater } \\
\text { Trochanter and } \\
\text { Lateral Epicondyle }\end{array}$ \\
\hline
\end{tabular}

Rest periods were offered to each subject between configuration tests. Walking and standing with the hips coupled was evaluated qualitatively in the able-bodied subject, to assure general compatibility between the CSO and the bracing system.

The Upper Torso, Orthosis, and Thighs were chosen as the body segments key to quantifying bending. These segments are defined by the marker placements listed in Table 1. For the No Brace configuration the Orthosis markers are placed on the lower torso as defined in Table 1. For CSO trials the CSO Bottom section was also analyzed as a separate segment from the Orthosis, or CSO top section. Subjects were asked to keep the lower body stationary, and though the thighs did move with the torso in some instances, the legs were consistently held together to create a single Thighs segment. At least three nonlinear markers at each segment were used to define a plane in 3D space (Fig. 12). Markers were placed at each wrist only as a visual aid to outline the body during analysis.

\subsection{Angle calculation method}

The ground coordinate system for the motion capture test volume was created with the same orientation as the seated surface; essentially in line with the principal axes of the upright seated subject. Matlab (Mathworks, Natick MA, USA) was used to perform movement analysis based on the xyz coordinates from each marker. For each segment, a system of three orthogonal axes was created using normalized vectors and cross products from three marker coordinates (Fig. 13). Any three markers were suitable, and if three markers were not present analysis was skipped for that particular frame. Dropped frames were rare and not expected to impact results, since only the two frames

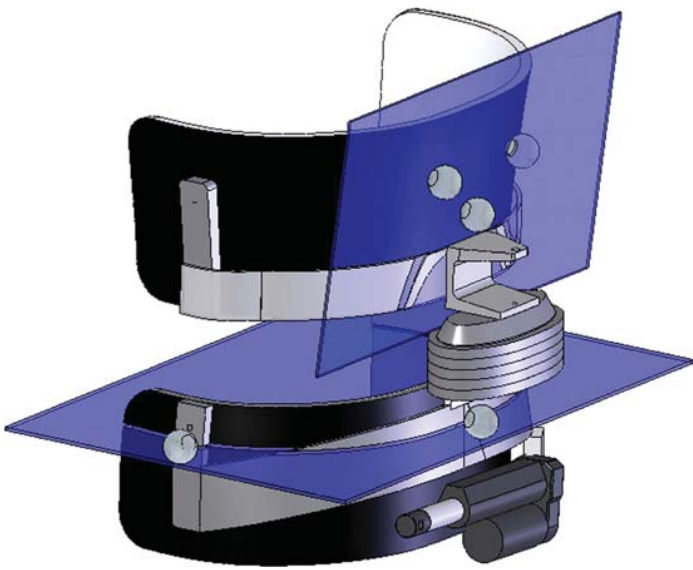

Fig. 12. Example marker placements and resulting planes used to define segment rotation based on the location of 3 or more markers.

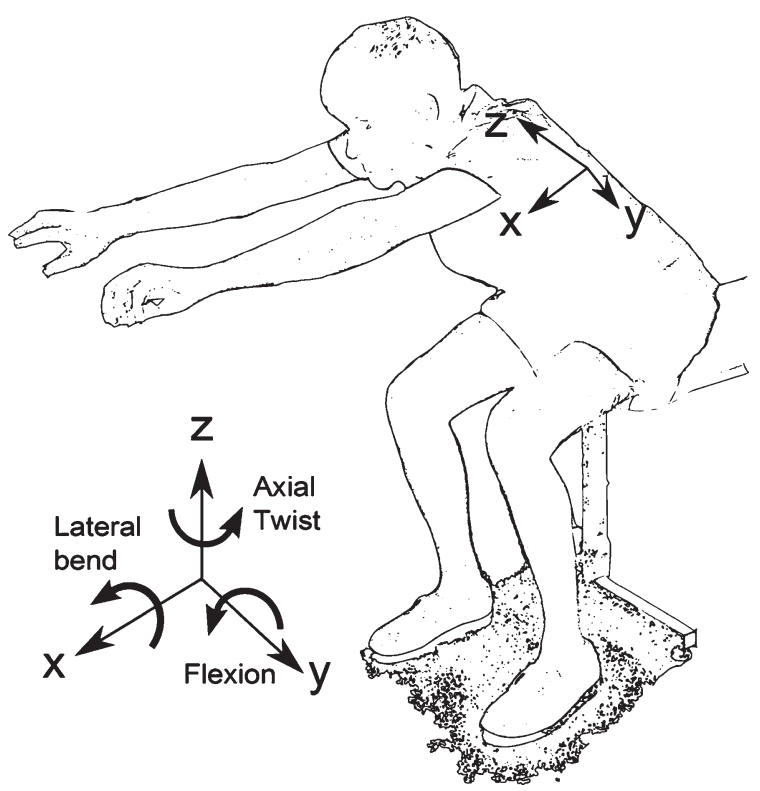

Fig. 13. The principal directions are $\mathrm{z}$ : Axial Twist, $\mathrm{x}$ : Lateral Bend, and y: Flexion. The segment coordinate system would be aligned with the ground system when the subject is sitting up straight.

containing the minimum and maximum excursions were ultimately necessary.

Before analysis of each trial, a rotation matrix was calculated to map the first frame of each segment system to the ground system. This rotation was applied throughout all frames to redefine the segment coordinate systems. In this way the initial frame for each segment is aligned with the ground reference frame and approximately aligned with the anatomical planes. 
Then, a rotation matrix from one segment system with respect to another segment system was calculated using matrix left division, signifying the angle differences between the two coordinate systems and represented in the segment coordinate system. Translation between coordinate systems was not calculated.

Initially, bending in all directions was combined into a single value using axis angle representation. This value was intended to signify the total amount of bending in the specified direction, under the assumption that bending in other directions would be minimal. In reality, bending motions did not occur solely along each principal direction and axis angle representation caused inflation of values, so Euler angles are presented instead. Euler angles (ZXY) were extracted from the rotation matrices. The zxy Euler angles were found using Matlab to convert the rotation matrices to quater- nions, and then extracting the Euler angles, to minimize gimbal lock.

\section{Results}

The average and standard deviation for maximum angles along the reported principal directions are reported in Table 2. Measurements for the Torso and Orthosis segments are presented relative to the Thighs and not Ground, in order to depict how the device constrains the body, instead of how the body moves as a whole. Forward Flexion and Downward Flexion results give less insight into torso movements and CSO function since these movements could be carried out from the hips alone, therefore only the principal direction data in Table 2 are reported for these trials.

Table 2

Angle excursion in degrees along the principal direction of every trial

\begin{tabular}{|c|c|c|c|c|c|c|c|c|c|c|}
\hline & & & \multicolumn{2}{|c|}{ Rigid } & \multicolumn{2}{|c|}{ Locked } & \multicolumn{2}{|c|}{ Unlocked } & \multicolumn{2}{|c|}{ None } \\
\hline & & & Mean & $\mathrm{SD}$ & Mean & $\mathrm{SD}$ & Mean & $\mathrm{SD}$ & Mean & $\mathrm{SD}$ \\
\hline \multirow{8}{*}{ 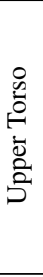 } & LB & $\mathrm{S}$ & 26.6 & 12.5 & 37.6 & 2.9 & 48.2 & 2.1 & 47.8 & $\overline{6.1}$ \\
\hline & & A & 21.8 & 1.9 & 26.1 & 1.5 & 48.1 & 5.2 & 45.0 & 1.7 \\
\hline & AT & $\mathrm{S}$ & 33.2 & 6.4 & 35.9 & 5.2 & 35.3 & 9.9 & 54.9 & 6.0 \\
\hline & & A & 57.1 & 2.5 & 47.5 & 4.7 & 57.4 & 2.6 & 58.4 & 13.7 \\
\hline & DF & $\mathrm{S}$ & 60.7 & 3.6 & 65.0 & 6.5 & 66.2 & 3.4 & 51.6 & 10.0 \\
\hline & & A & 74.0 & 1.9 & 28.5 & 3.4 & 25.9 & 8.2 & 29.4 & 4.1 \\
\hline & $\mathrm{FF}$ & $\mathrm{S}$ & 78.6 & 4.3 & 79.7 & 8.0 & 79.3 & 5.0 & 60.1 & 11.1 \\
\hline & & A & 90.5 & 4.8 & 36.3 & 2.2 & 39.7 & 4.2 & 64.3 & 8.7 \\
\hline \multirow{8}{*}{$\begin{array}{l}\text {. } \\
0 \\
\stackrel{0}{0} \\
0 \\
0\end{array}$} & LB & $\mathrm{S}$ & 7.0 & 1.6 & 17.3 & 1.4 & 36.5 & 1.6 & 52.0 & $\overline{6.4}$ \\
\hline & & A & 1.4 & 0.2 & 7.3 & 2.3 & 25.7 & 3.4 & 39.8 & 3.5 \\
\hline & AT & $\mathrm{S}$ & 1.4 & 0.6 & 13.9 & 2.8 & 15.6 & 4.3 & 48.8 & 6.6 \\
\hline & & A & 0.4 & 0.1 & 4.8 & 0.4 & 14.7 & 0.8 & 38.2 & 5.9 \\
\hline & DF & $\mathrm{S}$ & 51.6 & 3.2 & 52.1 & 9.1 & 65.4 & 1.3 & 60.5 & 14.1 \\
\hline & & A & 46.9 & 3.0 & 39.8 & 2.5 & 49.1 & 3.4 & 42.1 & 2.8 \\
\hline & FF & $\mathrm{S}$ & 59.1 & 3.3 & 59.3 & 12.9 & 69.3 & 4.3 & 85.6 & 17.3 \\
\hline & & A & 60.5 & 2.2 & 47.4 & 1.4 & 56.5 & 3.4 & 71.3 & 1.7 \\
\hline \multirow{8}{*}{$\frac{\mathscr{n}}{\frac{0}{D}}$} & LB & $\mathrm{S}$ & 11.7 & 1.7 & 14.1 & 1.7 & 12.5 & 2.0 & 9.5 & 1.2 \\
\hline & & A & 5.1 & 0.7 & 4.1 & 1.5 & 5.9 & 1.5 & 15.7 & 0.5 \\
\hline & AT & $\mathrm{S}$ & 1.1 & 0.7 & 1.1 & 0.3 & 1.6 & 0.7 & 2.2 & 0.9 \\
\hline & & A & 10.3 & 2.2 & 3.6 & 0.9 & 5.0 & 1.5 & 27.2 & 12.1 \\
\hline & DF & $\mathrm{S}$ & 5.7 & 0.8 & 6.1 & 1.6 & 6.9 & 0.7 & 17.4 & 5.4 \\
\hline & & A & 8.6 & 0.9 & 6.7 & 0.3 & 8.2 & 0.2 & 28.8 & 1.4 \\
\hline & $\mathrm{FF}$ & $\mathrm{S}$ & 5.9 & 0.6 & 7.2 & 1.3 & 7.1 & 0.5 & 20.3 & 5.1 \\
\hline & & A & 9.9 & 1.1 & 6.4 & 0.3 & 7.8 & 0.7 & 26.7 & 2.4 \\
\hline \multirow{8}{*}{ 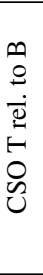 } & LB & $\mathrm{S}$ & $\mathrm{n} / \mathrm{a}$ & $\mathrm{n} / \mathrm{a}$ & 20.6 & 4.8 & 34.9 & 1.2 & $\mathrm{n} / \mathrm{a}$ & $\mathrm{n} / \mathrm{a}$ \\
\hline & & A & $\mathrm{n} / \mathrm{a}$ & $\mathrm{n} / \mathrm{a}$ & 4.2 & 0.7 & 24.9 & 1.1 & $\mathrm{n} / \mathrm{a}$ & $\mathrm{n} / \mathrm{a}$ \\
\hline & AT & $\mathrm{S}$ & $\mathrm{n} / \mathrm{a}$ & $\mathrm{n} / \mathrm{a}$ & 9.0 & 1.5 & 13.9 & 2.9 & $\mathrm{n} / \mathrm{a}$ & $\mathrm{n} / \mathrm{a}$ \\
\hline & & A & $\mathrm{n} / \mathrm{a}$ & $\mathrm{n} / \mathrm{a}$ & 5.3 & 0.2 & 15.5 & 0.8 & $\mathrm{n} / \mathrm{a}$ & $\mathrm{n} / \mathrm{a}$ \\
\hline & DF & $\mathrm{S}$ & $\mathrm{n} / \mathrm{a}$ & $\mathrm{n} / \mathrm{a}$ & 20.0 & 3.9 & 26.9 & 2.5 & $\mathrm{n} / \mathrm{a}$ & $\mathrm{n} / \mathrm{a}$ \\
\hline & & A & $\mathrm{n} / \mathrm{a}$ & $\mathrm{n} / \mathrm{a}$ & 3.9 & 0.3 & 7.8 & 1.4 & $\mathrm{n} / \mathrm{a}$ & $\mathrm{n} / \mathrm{a}$ \\
\hline & $\mathrm{FF}$ & $\mathrm{S}$ & $\mathrm{n} / \mathrm{a}$ & $\mathrm{n} / \mathrm{a}$ & 19.7 & 3.9 & 28.1 & 4.1 & $\mathrm{n} / \mathrm{a}$ & $\mathrm{n} / \mathrm{a}$ \\
\hline & & A & $\mathrm{n} / \mathrm{a}$ & $\mathrm{n} / \mathrm{a}$ & 7.2 & 0.3 & 12.5 & 0.5 & $\mathrm{n} / \mathrm{a}$ & $\mathrm{n} / \mathrm{a}$ \\
\hline
\end{tabular}

Configurations are listed in columns, while rows are arranged according to Segment, Movement (LB-Lateral Bend, AT-Axial Twist, DFDownward Flexion, FF-Forward Flexion), then Able-bodied (A) or SCI (S) data. 


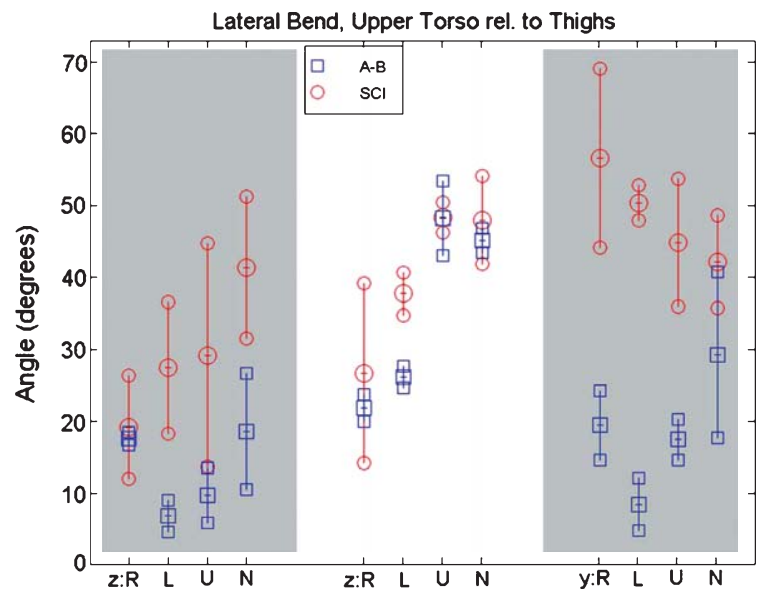

Fig. 14. Maximum excursion of the Upper Torso with respect to Thighs during Lateral Bend in the $\mathrm{z}$ (axial twist), $\mathrm{x}$ (lateral bend), and $y$ (flexion) directions. Movements about the principal $\mathrm{x}$ direction are not shaded. Averages across trials are plotted \pm one SD for each direction and configuration: Rigid (R), Locked (L), Unlocked (U), and No Brace $(\mathrm{N})$.

Maximum angle excursions in all directions for Lateral Bend and Axial Twist are plotted throughout the following sections, where un-shaded data represents the principal direction of motion for each movement. Maximum Euler angle excursion is calculated as the maximum value minus the minimum value throughout the movement, and is plotted \pm one standard deviation (SD).

\subsection{Upper Torso Motion with respect to Thighs}

In Lateral Bend, the Upper Torso segment (Fig. 14) does not exhibit pure bending along the $\mathrm{x}$-axis for either subject and is accompanied by sizeable forward flexion and axial twist. SCI ROM is consistently higher in both $\mathrm{x}$-axis principal rotation and $\mathrm{y}$ and $\mathrm{z}$ rotation, conceivably due to inability to control the body weight against gravity. Along the $\mathrm{x}$-axis, the able-bodied subject achieved similar ROM for the Rigid and Locked and also the Unlocked and No Brace configurations, approximately $25^{\circ}$ and $50^{\circ}$, respectively. This would indicate ideal device function. However, the SCI subject was able to push against the device resistance in the Locked state and achieve about $15^{\circ}$ more Locked than Rigid movement.

In Axial Twist (Fig. 15), the movement occurs mainly in the principal direction along the segment $\mathrm{z}$-axis without much movement in other directions. Except

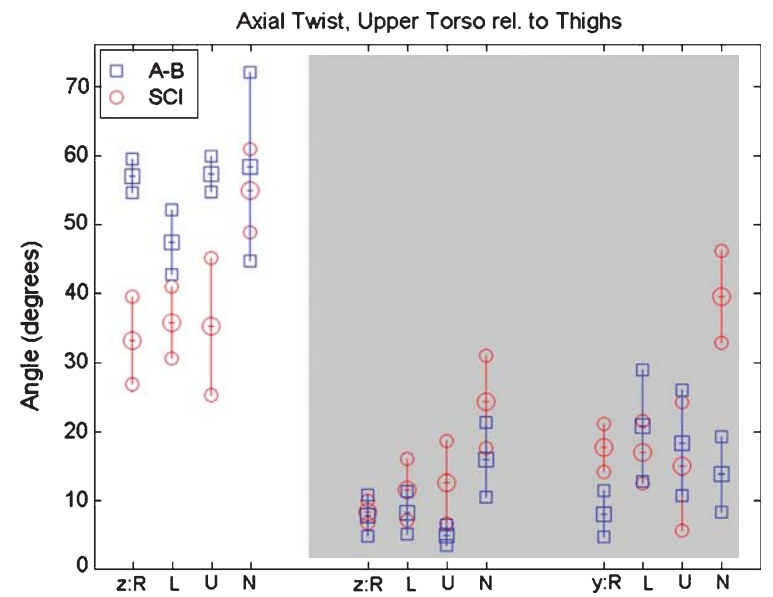

Fig. 15. Maximum excursion of the Upper Torso with respect to Thighs during Axial Twist in the $\mathrm{z}$ (axial twist), $\mathrm{x}$ (lateral bend), and $\mathrm{y}$ (flexion) directions. Movements about the principal $\mathrm{z}$ direction are not shaded. Averages across trials are plotted \pm one SD for each direction and configuration: Rigid (R), Locked (L), Unlocked (U), and No Brace $(\mathrm{N})$.

in the No Brace case, the able-bodied subject regularly displayed more z-axis ROM, approximately $55^{\circ}$ relative to $35^{\circ}$ in the SCI subject, most likely due to increased core strength and control. This subject used a noticeable amount of arm strength for stability in the No Brace configurations, which is illustrated by a drastic increase in forward bending during Axial Twist.

The SCI subject exhibited increased ROM in both the Forward Flexion and Downward Flexion configurations (Table 2); up to $40^{\circ}$ more than the able-bodied subject, with a relatively small difference between all bracing configurations and a slight decrease without bracing. It is interesting to note this relatively large amount of able-bodied bending while wearing the rigid orthosis during both Forward Flexion and Downward Flexion. A larger moment arm from the rigid orthosis is a potential cause, especially considering the unrestricted bending at the hip in this direction.

\subsection{Orthosis Motion with respect to Thighs}

Orthosis segment data for Lateral Bend are presented in Fig. 16. As expected, significantly more ROM was measured along the $\mathrm{x}$-axis for the Upper Torso as compared to the Orthosis, except with No Brace. As an exception the Orthosis actually experienced more bending than the Upper Torso without bracing since because both subjects kept their heads and shoulders relatively vertical as they bent. 


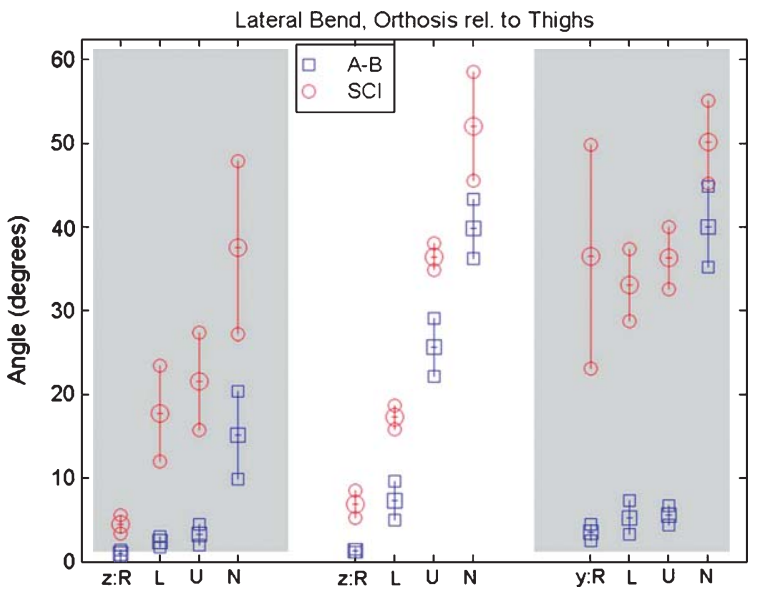

Fig. 16. Maximum excursion of the Orthosis with respect to Thighs during Lateral Bend in the $\mathrm{z}$ (axial twist), $\mathrm{x}$ (lateral bend), and $\mathrm{y}$ (flexion) directions. Movements about the principal x direction are not shaded. Averages across trials are plotted \pm one SD for each direction and configuration: Rigid (R), Locked (L), Unlocked (U), and No Brace $(\mathrm{N})$.

Comparing the Orthosis movement between the two subjects for Lateral Bend, the ROM along the x-axis for the SCI subject is consistently about $6^{\circ}-12^{\circ}$ higher, but the trend is the same across all configurations. In the Rigid state almost no motion is possible, while the CSO is Locked some amount of leeway is present, in the Unlocked state more motion is possible though it is constrained, and the No Brace state offers the maximum possible ROM. While performing the Lateral Bend the SCI subject also exhibits a significant amount of forward flexion and axial twist. The able-bodied subject constrains movement to almost pure lateral bending except in the No Brace configuration, which may have been possible from resistance feedback while wearing an orthosis.

During Axial Twist (Fig. 17) the configuration state has a large impact on Orthosis ROM about the $\mathrm{z}$-axis, with small increases between configuration states and a huge jump with no orthosis. This is in stark contrast with the nearly equivalent angles of twist across configurations for the Upper Torso segment (Fig. 15). The difference between maximum angles of the Upper Torso and Orthosis segments is high for both subjects in all three brace configurations and reaches a maximum above $50^{\circ}$ for the able-bodied subject in the Rigid orthosis. It should be noted that for all bracing configurations the Orthosis segment represents the brace, while in the No Brace configuration the Orthosis segment represents the lower torso directly.

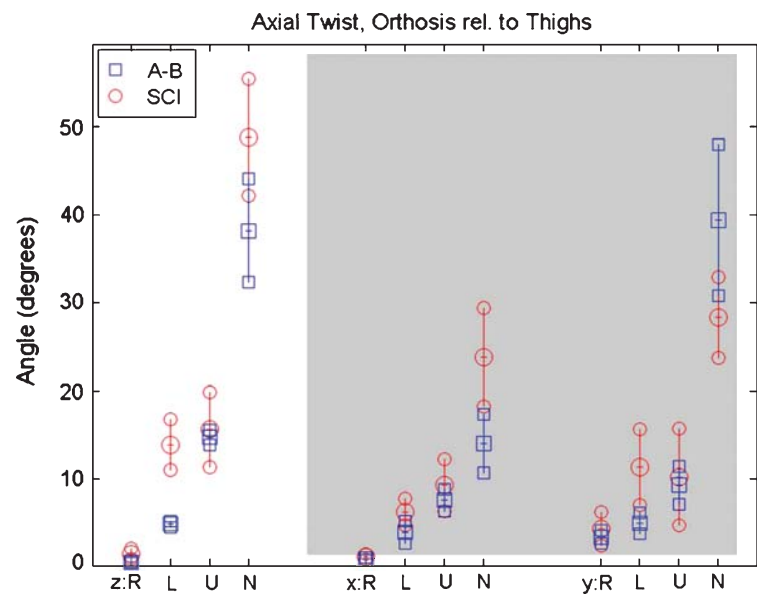

Fig. 17. Maximum excursion of the Orthosis with respect to Thighs during Axial Twist in the $\mathrm{z}$ (axial twist), $\mathrm{x}$ (lateral bend), and $\mathrm{y}$ (flexion) directions. Movements about the principal $\mathrm{z}$ direction are not shaded. Averages across trials are plotted \pm one SD for each direction and configuration: Rigid (R), Locked (L), Unlocked (U), and No Brace $(\mathrm{N})$.

Data from the SCI subject for the Forward Flexion movement are similar for the Upper Torso and Orthosis segments (Table 2). Able-bodied ROM, however, is significantly lower for the Upper Torso except in the Rigid configuration. This could have resulted from a desire to look at the arms as they reached forward, which would mean extending through the upper back and bringing the shoulders closer to vertical. For Downward Flexion there is little difference between the able-bodied and SCI subjects, or between either of the upper body segments. The different restrictions on torso motion for each configuration would have little impact if bending is predominantly from the hips.

\subsection{Thighs Motion with respect to Ground}

Examining the movement of the lower body is useful for understanding how the subject attempts to compensate for added constraints. Lateral Bend data from the thigh markers, analyzed as a single Thighs Segment, are shown in Fig. 18. During this movement, there is a tendency for the Thighs segment of both subjects and especially the SCI subject to bend forward about the $y$-axis as bending increases about the x-axis. The SCI subject displays more Lateral Bend of the Thighs in general through all of the bracing trials, which is probably a result of this subject using arm strength to throw the entire body into the movement. 


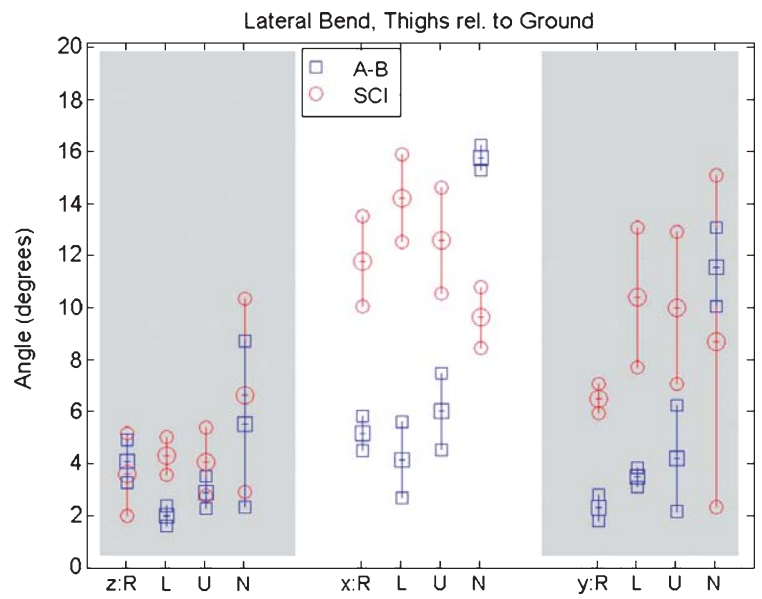

Fig. 18. Maximum excursion of the thighs with respect to Ground during Lateral Bend in the $\mathrm{z}$ (axial twist), $\mathrm{x}$ (lateral bend), and $\mathrm{y}$ (flexion) directions. Movements about the principal $\mathrm{x}$ direction are not shaded. Averages across trials are plotted \pm one SD for each direction and configuration: Rigid (R), Locked (L), Unlocked (U), and No Brace $(\mathrm{N})$.

The Thigh segments remained relatively still for both subjects during Axial Twist (Fig. 19), except during two of the four No Brace trials. In these exceptions the able-bodied subject seemed to use arm strength to twist the entire body, including the Legs, instead of twisting through the torso. Since body segment movements were calculated with respect to the Thighs this would not inflate other segment results. The Thighs segments for both subjects also remained stationary with less than $10^{\circ}$ of movement during Forward Flexion and Downward Flexion (Table 2). The No Brace configurations were again an exception for both subjects, with $17^{\circ}$ to $29^{\circ}$ of Thigh rotation as each subject bent forward enough to begin leaning out of the seat.

\subsection{Motion of Top relative to Bottom Portion of CSO}

The effect of movements on the CSO device can be assessed by examining the difference in excursion possible between the top and bottom portions in the Locked and Unlocked states. The Locked excursion represents the amount of mechanical play in the system, while the Unlocked excursion characterizes the maximum range of the combined wearer and device.

For movement of the top portion of the CSO relative to the bottom in the Unlocked state, the able-bodied subject achieved approximately $25^{\circ}$ in Lateral Bend (Fig. 20), $16^{\circ}$ in Axial Twist (Fig. 21), $8^{\circ}$ in Forward

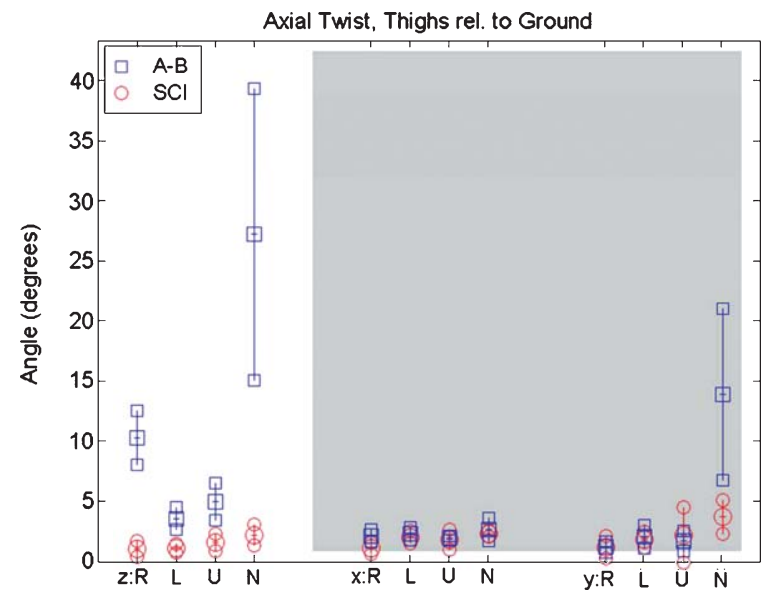

Fig. 19. Maximum excursion of the Thighs with respect to Ground during Axial Twist in the $\mathrm{z}$ (axial twist), $\mathrm{x}$ (lateral bend), and $\mathrm{y}$ (flexion) directions. Movements about the principal $\mathrm{z}$ direction are not shaded. Averages across trials are plotted \pm one SD for each direction and configuration: Rigid (R), Locked (L), Unlocked (U), and No Brace $(\mathrm{N})$.

Flexion, and $12^{\circ}$ in Downward Flexion (Table 2). The SCI subject attained less Axial Twist at $14^{\circ}$ (Fig. 21), but more range in Lateral Bend (Fig. 20), Forward Flexion, and Downward Flexion (Table 2) at $35^{\circ}, 28^{\circ}$, and $28^{\circ}$, respectively. The Lateral Bend values relate well to the amount of bending of the Orthosis segment relative to the Thighs (Fig. 16). ROM increases in order from the Locked to Unlocked configurations along all principal directions, as expected.

Ideally in the Locked state the angle between the top and bottom portions of the CSO would be zero. Due to a number of factors that will be elaborated upon, the Locked configuration was pushed by the SCI subject to a maximum across all trials of $20^{\circ}$ for Lateral Bend. All angle excursions in the Locked configuration were much greater with the SCI subject; the able-bodied subject never attained more than $7^{\circ}$ in any direction while the CSO was locked. This discrepancy is potentially due to the fact that the able-bodied subject did not push as far as possible after detecting resistance, whereas the SCI subject would be less capable of actively resisting gravity and have more potential to force the CSO to its limits.

\section{Discussion}

General trends emerge from the data. As shown by the averages and standard deviations, there are obvious 


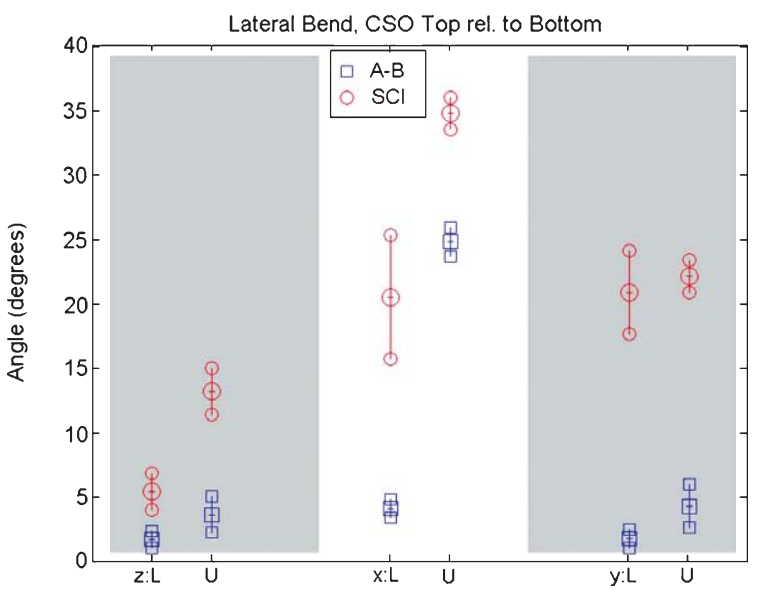

Fig. 20. Maximum excursion of Top relative to Bottom of CSO during Lateral Bend in the $\mathrm{z}$ (axial twist), $\mathrm{x}$ (lateral bend), and $\mathrm{y}$ (flexion) directions. Movements about the principal $\mathrm{x}$ direction are not shaded. Averages across trials are plotted \pm one SD for each direction and configuration: Rigid (R), Locked (L), Unlocked (U), and No Brace $(\mathrm{N})$.

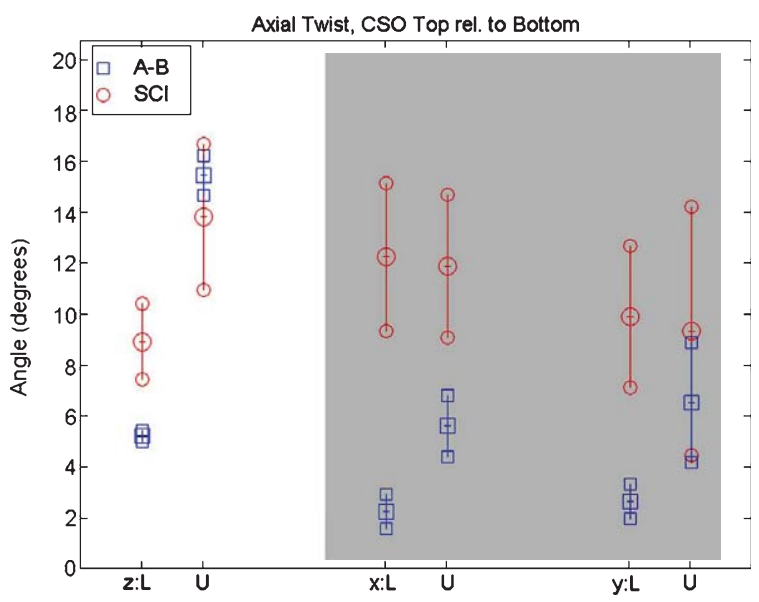

Fig. 21. Maximum excursion of Top relative to Bottom of CSO during Axial Twist in the $\mathrm{z}$ (axial twist), $\mathrm{x}$ (lateral bend), and $\mathrm{y}$ (flexion) directions. Movements about the principal $\mathrm{z}$ direction are not shaded. Averages across trials are plotted \pm one SD for each direction and configuration: Rigid (R), Locked (L), Unlocked (U), and No Brace $(\mathrm{N})$.

differences in ROM for the able-bodied and SCI subjects, with a few exceptions such as Lateral Bend of the Upper Torso segment. The able-bodied subject had a tendency to achieve more ROM in situations involving core strength, such as Axial Twist of the Upper Torso, while the SCI subject tended to display higher ROM in situations where gravity could aid motion, as in Lateral Bend or Forward Bending. The SCI subject was more liable than the able-bodied subject to move the entire body, as evidenced by increased tilt of the Thighs segment during Lateral Bend.

The Downward Flexion and Forward Flexion movements were least affected by changes in configuration state. This is likely due to the fact that the hip reciprocator was uncoupled, which essentially created a hinge joint in the sagittal plane allowing the user to flex freely at the hips, eliminating the need for flexion of the torso. In contrast, the hips were constrained laterally, and decreased orthosis constraints led to increased ROM of both the Upper Torso and Orthosis segments. The difference in maximum Axial Twist between configurations is a function of how well the orthosis is fitted to the body. The unlocked CSO would not provide a significant increase in twisting ability over the freedom already available if the torso can slide and rotate within the rigid or locked orthosis, which could potentially be prevalent even with a properly fitted orthosis.

There are inherent differences in the kinematics of a paraplegic and able-bodied subject performing a specific movement. These experiments were set up so that the No Brace configuration could be a control variable in comparing movements and interventions for each subject. While direct comparisons between the subjects are not necessarily appropriate, there are interesting hypotheses for the differences that do occur. An SCI subject may lack the proprioception necessary to maintain bending solely along a principal direction, which could lead to increased bending about multiple axes. Weak control of trunk muscles could either lead to a decrease in ROM if there is difficulty driving the movement, or an increase in ROM if gravity is acting on the body with no resistance from the core. An SCI subject with a T-7 level injury would rely on arm strength for positioning. An able-bodied subject might reduce effort when resistance from the device is felt, while an SCI subject would not necessarily detect or react to resistance.

\subsection{Unlocked state}

While ROM of the Upper Torso segment was approximately equal for the Unlocked and No Brace configurations, there was a decrease for the Orthosis except in Bending Forward. Limitations in the Unlocked state were particularly clear in Lateral Bend and Axial Twist and range could be improved for these configurations. Geometric limitations of the Unlocked CSO are a function of plate geometry and cable travel. 
Theoretically all constraints would be removed and the device behavior would converge to that of the No Brace configuration as plate size decreased, the number of plates increased, and actuator stroke increased. The decision for a smaller width than length was made to minimize the rear profile. All dimensions could be optimized further, especially if accuracy in control can be improved. Implementing an actuator with increased stroke length may be a simple step towards enhanced Unlocked ROM.

\subsection{Locked state}

Cable stretch, plate material compliance, and actuator inconsistencies are all factors in allowing mechanical play in the Locked configuration. Actuator position control is especially important for proper locking since as long as the cable reaches maximum length consistently, cable length can be tuned over time to account for plate elasticity and cable stretch. The linear actuator used in this device was predicted to have good position characteristics since the internal acme screw is not backdriveable. However, the limit switches do not adequately enforce position control. While switching to the Locked state, the moving end was observed to hit the limit switch and bounce back towards the Unlocked state, drifting up to $5 \mathrm{~mm}$ from the ideal stop.

The illustration from Fig. 10 can be used to analyze the effect of actuator drift on bending range. The error can be found by replacing the cable slack s with the drift distance and applying Equation 1. For actuator drift of $e=5 \mathrm{~mm}$, and radius of half the plate width $r=66.5 \mathrm{~mm}, 4.3^{\circ}$ of backlash would be possible for Lateral Bend in an ideal system. In reality, rounded edges of the plates and material compliance allow an even greater amount of backlash in the device. Active position control would reduce the amount of play possible in the Locked configuration, and consistent positioning would allow more precise pre-tensioning.

\section{Conclusion}

The goal of the CSO is to constrain motion and act as a traditional spinal orthosis while Locked, and to allow freedom of movement for the wearer while Unlocked. Using the current prototype and measuring the motion of the Upper Torso, the ROM while wearing a Rigid orthosis was similar to that of the Locked CSO, and the
ROM without any bracing was similar to the Unlocked CSO, for both an able-bodied and SCI subject. While these data alone suggest successful device function; analysis of the motion between the top and bottom sections of the CSO shows that some movement is still possible in the Locked state, and constraints on movement are present in the Unlocked state. Locked rigidity and Unlocked freedom could be improved with optimal plate geometry and accurate position control. The existing CSO device is currently capable of either allowing reaching and upper torso movement, or providing stability during walking.

\section{Acknowledgments}

This work was supported in part by the Department of Veterans Affairs, Rehabilitation Research and Development under Grant B3463 R and in part by the Department of Defense under Grant PR043074.

\section{References}

[1] J. Cholewicki, K. Alvi, S.P. Silfies and J. Bartolomei, Comparison of motion restriction and trunk stiffness provided by three thoracolumbosacral orthoses (TLSOs), Journal of Spinal Disorders \& Techniques 16(5) (2003), 461-468.

[2] R.J. Farris, H.A. Quintero, T.J. Withrow and M. Goldfarb, Design and Simulation of a Joint-Coupled Orthosis for Regulating FES-Aided Gait, Presented at IEEE International Conference on Robotics and Automation (ICRA'09), 2009, 1916-1922.

[3] M. Goldfarb, K. Korkowski, B. Harrold and W. Durfee, Preliminary evaluation of a controlled-brake orthosis for FES-aided gait, IEEE Transactions on Neural Systems and Rehabilitation Engineering 11(3) (2003), 241.

[4] R. Jailani, M.O. Tokhi and S. Gharooni, Hybrid Orthosis: The technology for spinal cord injury, Journal of Applied Sciences 10(22) (2010), 2785

[5] A. Kangude, B. Burgstahler and W. Durfee, Engineering Evaluation of the Energy-Storing Orthosis FES Gait System, Presented at Annual International Conference of the IEEE Engineering in Medicine and Biology Society (EMBC'10), 2010, 5927-5930.

[6] K.R. Kaufman, S.E. Irby, J.W. Mathewson, R.W. Wirta and D.H. Sutherland, Energy-efficient knee-ankle-foot orthosis: A Case Study, Journal of Prosthetics and Orthotics (JPO) 8(3) (1996), 79.

[7] N.I. Kern, T.J. Majewski, R.J. Triolo, R. Kobetic and R.D. Quinn, A locking compliant device inspired by the anatomy of the spine, J Mech Des 131(1) (2009), 014501.

[8] R. Kobetic and E.B. Marsolais, Synthesis of paraplegic gait with multichannel functional neuromuscular stimulation, IEEE Transactions on Rehabilitation Engineering 2(2) (1994), 66. 
[9] R. Kobetic, E.B. Marsolais, R.J. Triolo, D.T. Davy, R. Gaudio and S. Tashman, Development of a hybrid gait orthosis: A Case Report, J Spinal Cord Med 26(3) (2003), 254.

[10] R. Kobetic, C.S. To, J.R. Schnellenberger, M.L. Audu, T.C. Bulea, R. Gaudio, G. Pinault, S. Tashman and R.J. Triolo, Development of hybrid orthosis for standing, walking, and stair climbing after spinal cord injury, J Rehabil Res Dev 46(3) (2009), 447.

[11] R. Konz, S. Fatone and S. Gard, Effect of restricted spinal motion on gait, J Rehabil Res Dev 43(2) (2006), 161.

[12] E.B. Marsolais, R. Kobetic, G. Polando, K. Ferguson, S. Tashman, R. Gaudio, S. Nandurkar and H.R. Lehneis, The Case Western Reserve University Hybrid Gait Orthosis, J Spinal Cord Med 23(2) (2000), 100.

[13] C.S. To, R. Kobetic, J.R. Schnellenberger, M.L. Audu and R.J. Triolo, Design of a variable constraint hip mechanism for a hybrid neuroprosthesis to restore gait after spinal cord injury,
IEEE/ASME Transactions on Mechatronics 13(2) (2008), 197.

[14] R.L. Waters, J. Campbell, L. Thomas, L. Hugos and P. Davis, Energy costs of walking in lower-extremity plaster casts, J Bone Joint Surg Am 64(6) (1982), 896.

[15] W.Y. Wong and M.S. Wong, Detecting spinal posture change in sitting positions with Tri-Axial accelerometers, Gait Posture 27(1) (2008), 168.

[16] M. Wunderlich, T. Rüther, D. Essfeld, T.C. Erren, C. Piekarski and D. Leyk, A new approach to assess movements and isometric postures of spine and trunk at the workplace, European Spine Journal (8) (2011), 1393.

[17] S. Zhang, K.G. Clowers, M. Wortley and J.H. Krusenklaus, Efficacy of lumbar and lumbosacral orthoses in restricting spinal ROMs, Journal of Back \& Musculoskeletal Rehabilitation 19(2) (2006), 49. 

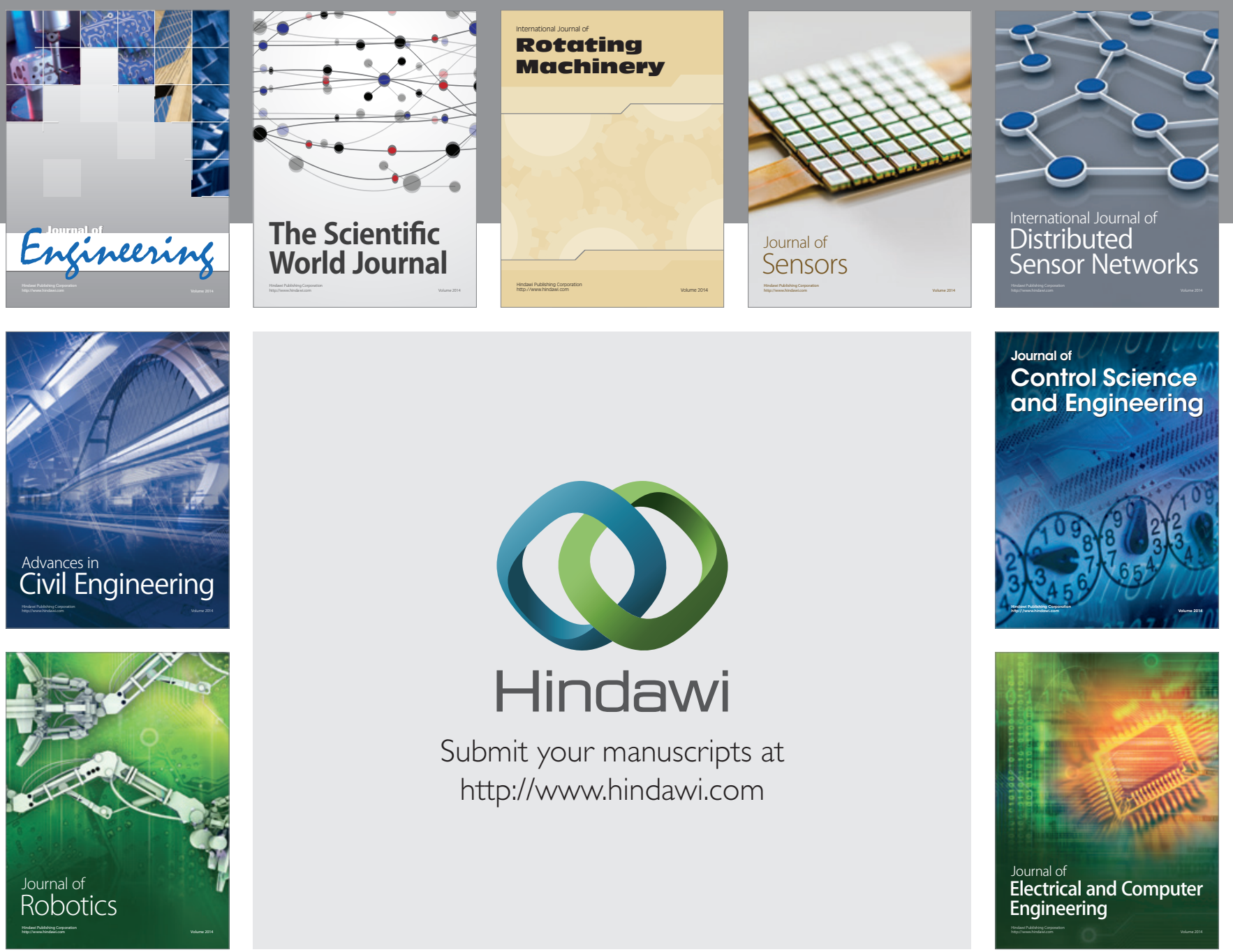

Submit your manuscripts at

http://www.hindawi.com
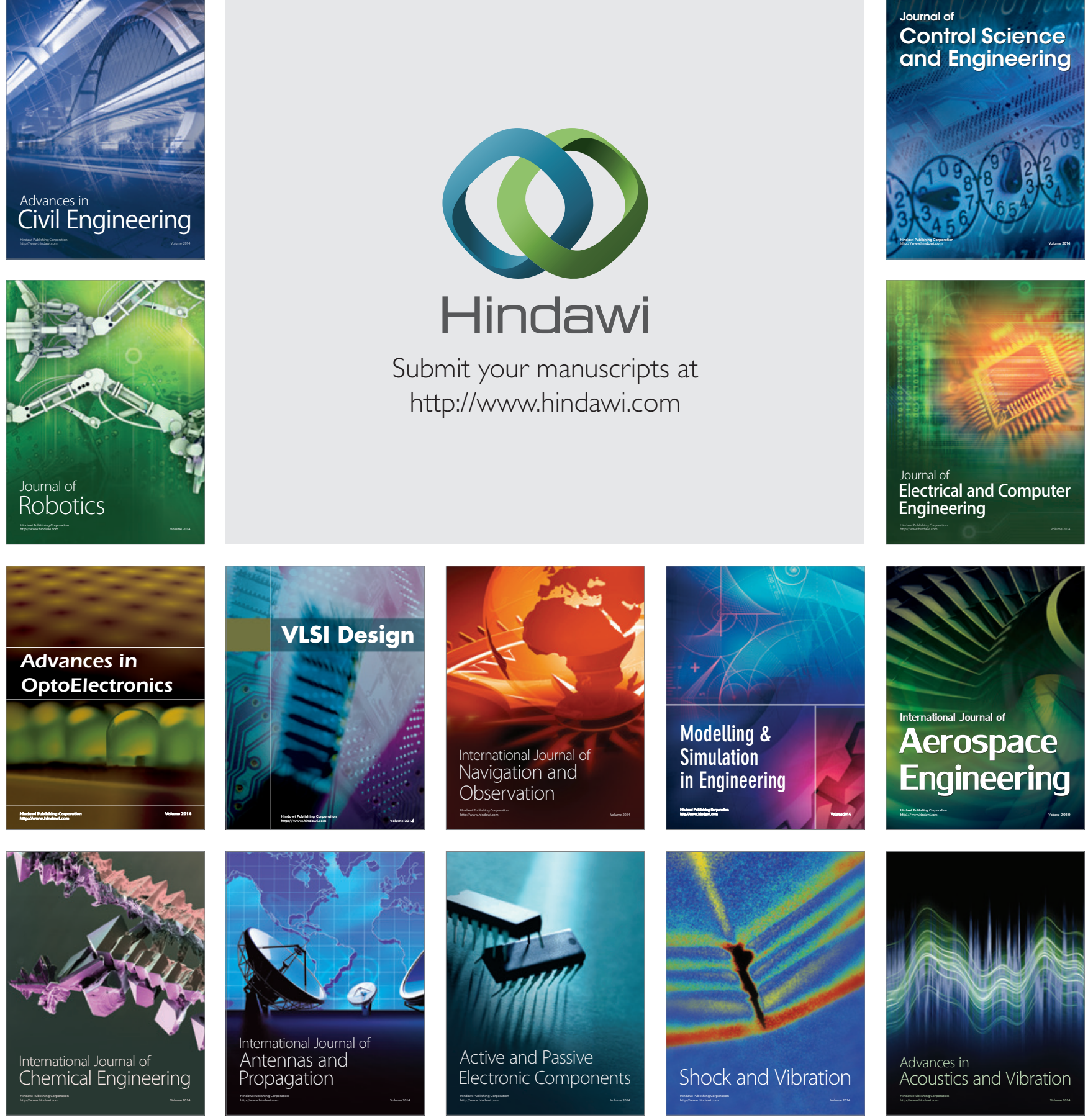